\title{
La diffusion quasiélastique des neutrons ; introduction et principes généraux
}

\author{
M. Bée
}

Laboratoire de Spectrométrie Physique, UMR 5588, Université Joseph Fourier Grenoble I, BP. 87, 38402 Saint-Martin-d'Hères cedex 9, France

\begin{abstract}
Résumé. Ce cours vise à introduire les principes fondamentaux de la diffusion quasiélastique incohérente de neutrons, en partant du niveau élémentaire des propriétés du neutron et de l'interaction neutron-noyau. Nous établissons d'abord les deux aspects différents de cette interaction, et nous séparons la fonction de diffusion en une partie cohérente et une partie incohérente. La très grande section efficace incohérente de l'hydrogène, comparée à celle des autres éléments, fait de la diffusion des neutrons une technique privilégiée pour l'analyse des mouvements individuels des protons dans les composés hydrogénés. Sous réserve de pouvoir négliger les interactions entre les différentes composantes du déplacement d'un diffuseur (vibrations, rotation ou translation), leurs contributions à l'intensité diffusée peuvent être séparées et il est possible d'accéder à la fonction de diffusion quasiélastique dont l'élargissement est directement reliée aux temps caractéristiques des mouvements sur la gamme de temps $10^{-111}$ $10^{-12} \mathrm{~s}$. La part de l'intensité diffusée purement élastique renseigne quant à elle sur la géométrie de ces mouvements.
\end{abstract}

\section{PROPRIETES DU NEUTRON}

Le neutron est une particule élémentaire de charge nulle, de masse $m=1,66010^{-27} \mathrm{~kg}$, de spin nucléaire $1 / 2$ et de moment magnétique $\mu=-1,913$ magnéton nucléaire. L'énergie, $E$, d'un neutron libre, de vitesse $\boldsymbol{v}$ est simplement égale à son énergie cinétique

$$
E=\frac{1}{2} m v^{2}
$$

Les neutrons peuvent aussi être considérés comme des ondes planes, de vecteur d'onde

$$
\boldsymbol{k}=\frac{m}{\hbar} \mathbf{v}
$$

et de longueur d'onde

$$
\lambda=\frac{2 \pi}{|\boldsymbol{k}|}=\frac{h}{m V}
$$

de sorte que l'énergie cinétique s'exprime comme

$$
E=\frac{1}{2} m v^{2}=\frac{\hbar^{2} k^{2}}{2 m}
$$

Les neutrons issus du cœur du réacteur nucléaire sont thermalisés en passant au travers d'un modérateur à la température $T$. Dès lors la distribution de leurs vitesses suit une loi de Maxwell autour d'une valeur moyenne $v$ telle que :

$$
E=\frac{1}{2} m v^{2}=\frac{3}{2} k_{B} T
$$

$k_{B}$ étant la constante de Boltzmann. Les neutrons thermiques, correspondant à $\mathrm{T}=300 \mathrm{~K}$, ont une énergie d'environ $25 \mathrm{meV}$ (milliélectronvolts) et une longueur d'onde de $1,8 \AA(v=2200 \mathrm{~m} / \mathrm{s})$. Les «sources froides 》 ou «sources chaudes» thermalisent les neutrons à $25 \mathrm{~K}$ ou $2000 \mathrm{~K}$ et permettent de déplacer le maximum de la distribution respectivement vers les grandes longueurs d'onde $(3,5 \AA)$ ou vers les courtes longueurs d'onde $(0,5 \AA)$. 
De ces valeurs on retiendra surtout que l'énergie des neutrons thermiques apparaît du même ordre de grandeur que les énergies intermoléculaires dans les phases condensées. En outre, leur longueur d'onde est comparable aux dimensions moléculaires usuelles et aux distances interréticulaires dans les solides. Ces deux propriétés font du neutron un outil très puissant pour l'étude microscopique des propriétés tant dynamiques que géométriques de la matière.

\section{DEFINITION DES SECTIONS EFFICACES DE DIFFUSION}

Quand un neutron passe près d'un noyau, deux éventualités peuvent se produire

i) le neutron est absorbé par le noyau, ou bien

ii) il subit une diffusion dans laquelle tant son énergie que sa direction peuvent changer.

L'énergie des neutrons froids ou thermiques est certes trop faible pour provoquer des excitations internes du noyau ou des couches électroniques. Néanmoins le neutron est sensible aux déplacements atomiques des noyaux qui correspondent à des énergies beaucoup plus faibles et qui sont à l'origine de la diffusion inélastique.

Les sections efficaces de diffusion ou d'absorption se définissent à partir du nombre de processus de diffusion, $I_{s}$, ou d'absorption, $I_{a}$ se produisant chaque seconde quand un faisceau de $I_{0}$ neutrons par centimètre carré et par seconde arrive sur un échantillon, suivant les relations :

$$
\begin{aligned}
& I_{s}=I_{0} \sigma_{s} \\
& I_{a}=I_{0} \sigma_{a}
\end{aligned}
$$

L'unité habituelle de $\sigma_{s}$ et $\sigma_{a}$ est le barn ( 1 barn $=10^{-24} \mathrm{~cm}^{2}$ ).

Aux faibles énergies de quelques $\mathrm{meV}$, la section efficace d'absorption d'un neutron par un noyau, $\sigma_{t}$ est proportionnelle à la longueur d'onde du neutron incident, $\lambda$ (soit à l'inverse de la vitesse, $v$ )

$$
\begin{gathered}
\sigma_{a} \propto \frac{1}{\lambda} \\
\sigma_{a} \propto \frac{1}{v}
\end{gathered}
$$

Un neutron interagit avec un noyau par l'intermédiaire de forces nucléaires et magnétiques. Comparée à la longueur d'onde des neutrons thermiques, $\left(10^{-10} \mathrm{~m}\right)$ la partie nucléaire de l'interaction est à très courte portée $\left(10^{-14}-10^{-15} \mathrm{~m}\right)$. Aussi la diffusion neutron - noyau ne fait-elle intervenir que des ondes de type $s$ : la diffusion est isotrope et se caractérise par un paramètre unique, la longueur de diffusion, $b$. Ce paramètre peut être complexe et varier rapidement avec l'énergie du neutron. La partie imaginaire de la diffusion correspond à une absorption. De tels noyaux absorbent fortement les neutrons, formant un noyau composé dont l'énergie est proche d'un état excité (ex: ${ }^{113} \mathrm{Cd},{ }^{157} \mathrm{Gd},{ }^{103} \mathrm{Rh},{ }^{173} \mathrm{Lu}$ ). Cependant dans la majorité des cas le noyau composé est loin d'un état excité. La partie imaginaire de la longueur de diffusion est petite et la longueur de diffusion est indépendante de l'énergie du neutron. Nous nous placerons dans cette situation et nous considérerons la longueur de diffusion comme une quantité réelle, positive ou négative selon la nature répulsive ou attractive de l'interaction.

La longueur de diffusion, $b$, change non seulement en passant d'une espèce chimique à l'autre, mais aussi pour les différents isotopes de la même espèce, car l'interaction neutron - noyau dépend non seulement de la nature de ce dernier mais aussi de l'état de spin total du système. Lors d'une expérience, l'échantillon est composé de différentes espèces atomiques, $i$, chacune d'elle pouvant être un mélange de plusieurs isotopes possédant leur propre spin nucléaire.

Pour une espèce chimique donnée, la moyenne $\overline{b_{i}}$ des longueurs de diffusion prise sur les différents isotopes et sur tous les états de spin est appelée la longueur de diffusion cohérente, $b_{i}{ }^{\text {coh }}$. Dans le même temps, l'écart quadratique moyen de $b_{i}$ à $\overline{b_{i}}$ définit la longueur de diffusion incohérente:

$$
\begin{gathered}
b_{i}^{c o h}=\overline{b_{i}} \\
b_{i}^{i n c}=\sqrt{\overline{b i}_{i}^{2}-{\overline{b_{i}}}^{2}}
\end{gathered}
$$


Les longueurs de diffusion cohérente et incohérente sont de nature profondément différente. La diffusion cohérente est celle à laquelle le système donnerait lieu si tous les atomes d'une même espèce avaient la même longueur de diffusion $\overline{b_{i}}$. Le neutron verrait alors un potentiel uniforme moyen, pouvant donner lieu à des phénomènes d'interférences. La diffusion incohérente est celle qui s'ajoute à la diffusion cohérente pour obtenir la diffusion du système réel. Les écarts au potentiel moyen étant distribués au hasard, ils ne peuvent être à l'origine de phénomènes d'interférences.

Le neutron ayant un spin $1 / 2$, pour un noyau de spin $s$ non nul, le spin du système neutron - noyau vaut soit $s+1 / 2$, soit $s-1 / 2$. Ainsi chaque noyau de spin non nul se voit associer deux valeurs de la longueur de diffusion. Seuls les noyaux de spin zéro conduisent à une seule longueur de diffusion, le système neutron - noyau pouvant avoir seulement le spin $1 / 2$.

Le cas le plus simple est celui d'un seul isotope, de spin nucléaire zéro. Alors toutes les longueurs de diffusion sont égales et la diffusion est entièrement cohérente.

Avec un seul isotope, de spin nucléaire s, il y a deux longueurs de diffusion $b^{+}$et $b^{-}$, associées aux deux états de spin $S^{+}=s+1 / 2$ et $S=s-1 / 2$ du système total neutron - noyau. Il y a respectivement $n^{+}=2 S^{+}+1$ et $n^{-}=2 S+1$ états de spin $S^{+}$et $S$. Si les neutrons ne sont pas polarisés et si les spins nucléaires sont distribués au hasard, chaque état de spin a la même probabilité de sorte que

$$
\begin{gathered}
\bar{b}=\frac{1}{n^{+}+n^{-}}\left[n^{+} b^{+}+n^{-} b^{-}\right] \\
b^{c o h}=\frac{1}{2 s+1}\left[(s+1) b^{+}+s b^{-}\right] \\
\overline{b^{2}}=\frac{1}{n^{+}+n^{-}}\left[n^{+}\left(b^{+}\right)^{2}+n^{-}\left(b^{-}\right)^{2}\right]
\end{gathered}
$$

Le cas général est celui où les différentes espèces atomiques présentes dans l'échantillon sont composées de plusieurs isotopes, $\alpha$, d'abondance relative $c_{\alpha}$.

$$
\begin{gathered}
\vec{b}=\sum_{\alpha} c_{\alpha} \frac{1}{2 s_{\alpha}+1}\left[\left(s_{\alpha}+1\right) b_{\alpha}^{+}+s_{\alpha} b_{\alpha}^{-}\right] \\
\overline{b^{2}}=\sum_{\alpha} \frac{c_{\alpha}}{2 s_{\alpha}+1}\left[\left(s_{\alpha}+1\right)\left(b_{\alpha}^{+}\right)^{2}+s_{\alpha}\left(b_{\alpha}^{-}\right)^{2}\right]
\end{gathered}
$$

Si les neutrons sont polarisés ou si les spins nucléaires sont alignés, les $2 s+2$ états de spin du système neutron - noyau ne sont plus équiprobables et les expressions précédentes des moyennes sur les états de spin ne sont plus valables. Certaines techniques instrumentales utilisent la polarisation des neutrons pour distinguer la diffusion cohérente de la diffusion incohérente. Néanmoins, pour éliminer la moyenne sur l'abondance relative des différents isotopes, il faut utiliser des espèces atomiques isotopiquement pures. Cette condition n'est pas toujours aisée à satisfaire.

Ces expressions de $\bar{b}$ et $\overline{b^{2}}$ permettent d'évaluer les sections efficaces dites de l'atome lié, à savoir la section efficace de diffusion incohérente,

$$
\sigma_{c o h}=4 \pi \bar{b}^{2}
$$

et la section efficace de diffusion incohérente

$$
\begin{aligned}
\sigma_{i n c} & =4 \pi\left(\overline{\left.b^{2}-\bar{b}^{2}\right)}\right. \\
& =4 \pi \overline{(b-\bar{b})^{2}}
\end{aligned}
$$


ainsi que la section efficace de diffusion totale

$$
\sigma_{t o t}=\sigma_{c o h}+\sigma_{i n c}=4 \pi \overline{b^{2}}
$$

Le proton unique de spin $1 / 2$ qui constitue le noyau de l'atome d'hydrogène en fait un cas particuliè intéressant. Les longueurs de diffusion sont:

$$
b^{+}=1,0810^{-12} \mathrm{~cm}
$$

pour l'état total triplet $S^{+}=1 / 2, n^{+}=3$, et

$$
b^{-}=-4,7410^{-12} \mathrm{~cm}
$$

pour l'état total singulet $S^{-}=0, n^{-}=1$. En appliquant les relations établies précédemment, nous obtenons

$$
\begin{aligned}
& \bar{b}=\frac{3}{4} b^{+}+\frac{1}{4} b^{-}=-0,3710^{-12} \mathrm{~cm} \\
& \overline{b^{2}}=\frac{3}{4}\left(b^{+}\right)^{2}+\frac{1}{4}\left(b^{-}\right)^{2}=6,4910^{-24} \mathrm{~cm}^{2}
\end{aligned}
$$

de sorte que les sections efficaces de diffusion, cohérente, incohérente et totale, exprimées en barns $\left(1\right.$ barn $\left.=10^{-24} \mathrm{~cm}^{2}\right)$ sont égales à

$$
\begin{aligned}
& \sigma_{\text {coh }}\left({ }^{1} \mathrm{H}\right)=1,8 \text { barns, } \\
& \sigma_{\text {inc }}\left({ }^{1} \mathrm{H}\right)=79,9 \text { barns, } \\
& \sigma_{\text {tot }}\left({ }^{1} \mathrm{H}\right)=81,7 \text { barns. }
\end{aligned}
$$

La différence très importante entre $\sigma_{c \cdot o h}\left({ }^{1} \mathrm{H}\right)$ et $\sigma_{i n c}\left({ }^{\mathrm{l}} \mathrm{H}\right)$ provient essentiellement des signes opposés de $b^{+}$et $b^{-}$. Nous donnons dans le tableau I une liste de quelques valeurs de $\sigma_{c u l h}$ et $\sigma_{i n c}$ pour différents éléments. D'une manière générale la section efficace de diffusion incohérente de l'hydrogène est beaucoup plus grande que celle de tous les autres éléments, ce qui fait de la diffusion incohérente des neutrons un outil privilégié pour l'étude des composés hydrogénés.

Table 1: Longueurs de diffusion et sections efficaces de quelques éléments

\begin{tabular}{lcccrrrrrr}
\hline & $\mathrm{Z}$ & $\mathrm{A}$ & \multicolumn{1}{c}{ Spin } & $b^{\text {coh }}\left(10^{-15} \mathrm{~m}\right)$ & $b^{i n c}\left(10^{-15} \mathrm{~m}\right)$ & $\sigma_{c o h}$ (barns) & $\sigma_{\text {inc }}$ (barns) & $\sigma_{a}$ (barns) \\
\hline $\mathrm{H}$ & 1 & 1 & $1 / 2$ & -3.742 & 25.217 & 1.760 & 79.91 & 0.3326 \\
& & 2 & 1 & 6.674 & 4.033 & 5.597 & 2.04 & 0.0005 \\
$\mathrm{C}$ & 6 & 12 & 0 & 6.653 & 0 & 5.563 & 0 & 0.0035 \\
$\mathrm{O}$ & 8 & 16 & 0 & 5.805 & 0 & 4.235 & 0 & 0.0002 \\
$\mathrm{Al}$ & 13 & 27 & $5 / 2$ & 3.449 & 0.26 & 1.495 & 0.01 & 0.231 \\
$\mathrm{~V}$ & 23 & 51 & $-7 / 2$ & -0.402 & 6.42 & 0.020 & 5.19 & 5.08 \\
\hline
\end{tabular}

Le cas du deutérium est très différent. Le spin nucléaire vaut $s=1$. Pour l'état $S^{+}=3 / 2, n^{+}=4$, la longueur de diffusion vaut

$$
b^{+}=0,9510^{-12} \mathrm{~cm},
$$

tandis que pour l'état $S=1 / 2, n^{-}=2$ elle est égale à

$$
b^{-}=0,1010^{-12} \mathrm{~cm} \text {. }
$$


Nous obtenons ainsi les sections efficaces de diffusion

$$
\begin{gathered}
\sigma_{c o h}\left({ }^{2} \mathrm{H}\right)=5,6 \text { barns } \\
\sigma_{\text {inc }}\left({ }^{2} \mathrm{H}\right)=2,0 \text { barns } \\
\sigma_{t o t}\left({ }^{2} \mathrm{H}\right)=7,6 \text { barns }
\end{gathered}
$$

La part relative de la diffusion incohérente dans la diffusion totale est cette fois considérablement réduite, par rapport au cas de l'hydrogène ${ }^{1} \mathrm{H}$. Ainsi lorsqu'on s'intéresse à la diffusion cohérente des neutrons par les composés organiques il vaut micux, chaque fois que c'est possible réaliser les expériences avec des composés deutériés. De leur coté, les mesures réalisées en diffusion incohérente des neutrons peuvent tirer avantage d'une deutération sélective partielle de l'échantillon, afin de faire varier les contributions respectives de différents groupes chimiques à l'intensité diffusée.

Pour les neutrons, les éléments lourds ou légers peuvent avoir des longueurs de diffusion comparables. C'est la situation inverse de celle des rayons X pour lesquels les facteurs de diffusion sont proportionnels au numéro atomique, de sorte que les éléments légers sont presque invisibles dans les mesures, particulièrement si des éléments lourds sont présents. Avec les neutrons, aucun obstacle ne s'oppose a priori à l'observation d'éléments légers, pourvu que leur longueur de diffusion soit suffisante.

Signalons pour conclure qu'en plus de l'hydrogène, d'autres éléments ont une importance particulière en diffusion neutronique. Avec une section efficace d'absorption $\sigma_{a}(\mathrm{Al})=0,231$ barn pour une longueur d'onde $\lambda=1,8 \mathrm{~A}$, l'aluminium absorbe très peu les faisceaux neutroniques ( $1 \%$ par millimètre d'épaisseur). Aussi est-il couramment utilisé pour la réalisation de conteneurs d'échantillons, de fenêtres de cryostats ou encore d'épaisses cellules pour les mesures sous haute pression. A l'inverse, le cadmium et plus encore le gadolinium absorbent très fortement $\left(\sigma_{a}(\mathrm{Cd})=2520\right.$ barns, $\sigma_{a}(\mathrm{Gd})=29400$ barns à $\lambda=1,8 \mathrm{~A}$ ). Ces matériaux sont utilisés pour fabriquer des écrans destinés à éliminer les réflexions parasites ou des fentes servant à délimiter la forme et la dimension des faisceaux. Le vanadium est un diffuseur presque purement incohérent $\left(\sigma_{\text {cosh }}(\mathrm{V})=0,03\right.$ barn et $\sigma_{\text {inc }}(\mathrm{V})=5,1$ barns $)$. Il sert souvent de matériau de référence pour l'étalonnage des instruments (efficacité relative des détecteurs, mesure de la résolution expérimentale).

\section{LES FONCTIONS DE DIFFUSION NEUTRONIQUES}

Le schéma général d'une expérience de diffusion de neutrons est représenté sur la figure 1.

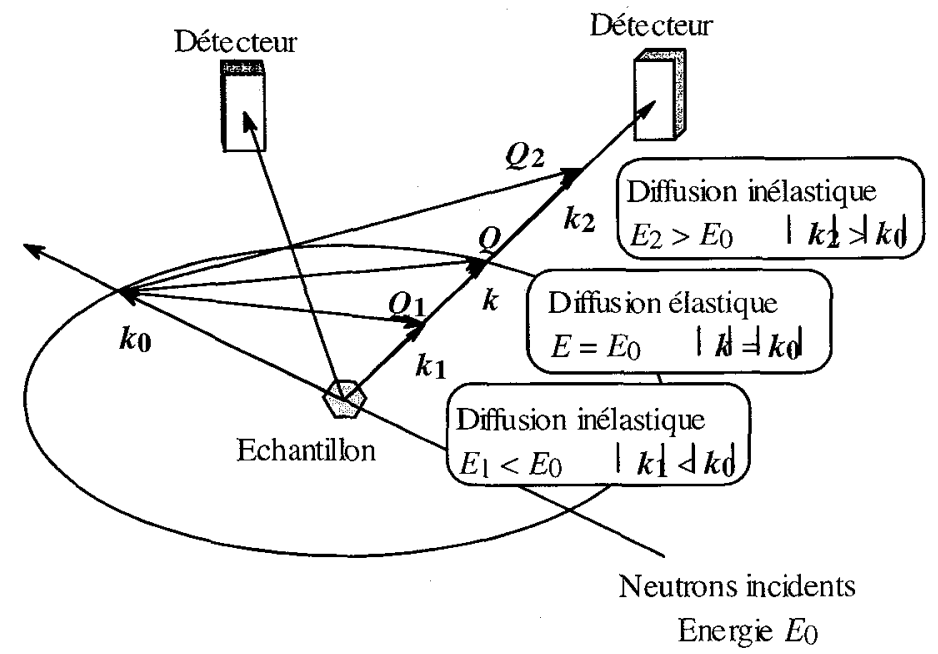

Figure 1: Schéma général d'une expérience de diffusion de neutrons. Pour une même valeur de l'angle de diffusion, le vecteur de diffusion, $Q$, varie en fonction de l'énergie échangée par le neutron avec l'atome diffuseur. 
Les deux principales grandeurs mesurées sont:

i) le vecteur de diffusion, $Q$, différence entre les vecteurs d'onde final, $k$, et initial, $\boldsymbol{k}_{\boldsymbol{\theta}}$ du neutron

$$
Q=k-k_{0}
$$

ii) la différence, $\hbar \omega$ entre les valeurs finales, $E$, et initiale, $E_{0}$, de l'énergie du neutron,

$$
\hbar \omega=E-E_{0}=\frac{\hbar^{2}}{2 m}\left(k^{2}-k_{0}^{2}\right)
$$

La formule de base de la diffusion nucléaire, donnant la probabilité qu'un neutron, arrivant sur un ensemble de $N$ diffuseurs avec l'énergie incidente $E_{0}$ et un vecteur d'onde $\boldsymbol{k}_{\mathbf{0}}$ soit diffusé avec le vecteur d'onde $k$ dans l'élément d'angle solide $d \Omega$ autour de la direction de $k$ et avec une énergie comprise entre $\mathrm{E}$ et $E+\hbar d \omega$ est

$$
\frac{d^{2} \sigma}{d \Omega d \omega}=\frac{k}{k_{0}} \frac{1}{N} \sum_{\alpha} \sum_{\beta} \frac{1}{2 \pi} \int_{\infty}^{\infty}\left\langle b_{\alpha} b_{\beta} \cdot e^{-i Q \cdot r_{\alpha}(0)} e^{i Q \cdot r_{\beta}(t)}\right\rangle e^{-i \omega t} d t
$$

La moyenne thermique porte non seulement sur les positions $\boldsymbol{r}_{\alpha}(0)$ et $\boldsymbol{r}_{\beta}(t)$ des noyaux de l'échantillon thais aussi sur tous les états de spin. En général, il n'existe pas de couplage entre la longueur de diffusion d'un noyau et sa position de sorte que les moyennes sur les états de spin et sur les coordonnées peuvent être prises séparément

$$
\frac{d^{2} \sigma}{d \Omega d \omega}=\frac{k}{k_{0}} \frac{1}{N} \sum_{\alpha} \sum_{\beta} \overline{b_{\alpha} b_{\beta}} \frac{1}{2 \pi} \int_{-\infty}^{\infty}\left\langle e^{-i Q \cdot r_{\alpha}(0)} e^{i Q \cdot r_{\beta}(t)}\right\rangle e^{-i \omega t} d t
$$

Cette expression dans laquelle la moyenne thermique ne porte plus que sur les coordonnées, est valable pour un système composé de plusieurs types de diffuseurs, les sommes sur les indices $\alpha$ et $\beta$ des atomes portant à la fois sur les différentes espèces chimiques et sur les isotopes de chaque espèce. En réalité, dans ce cours nous considérerons essentiellement la diffusion incohérente par des composés organiques hydrogénés. Les grandeurs fondamentales de la diffusion des neutrons peuvent s'introduire dans l'hypothèse d'un système à un seul composant. Pour plus d'information concernant le cas des systèmes à plusieurs composants, nous renvoyons à la littérature (Lovesey 1984, Squires 1978, Kostorz 1978). Signalons que

$$
\sigma=\int d \omega \int d \Omega \frac{\partial^{2} \sigma}{\partial \omega \partial \Omega}
$$

Nous avons défini précédemment les sections efficaces cohérentes et incohérentes relatives à un isotope, ce qui nous permet d'écrire l'équation précédente sous la forme

$$
\begin{gathered}
\frac{d^{2} \sigma}{d \Omega d \omega}=\frac{k}{k_{0}} \bar{b}^{2} \frac{1}{N} \sum_{\alpha} \sum_{\beta} \frac{1}{2 \pi} \int_{\infty}^{\infty}\left\langle\cdot e^{-i Q \cdot r_{\alpha}(0)} e^{i Q \cdot r_{\beta}(t)}\right\rangle e^{-i \omega t} d t \\
+\frac{k}{k_{0}}\left(\overline{b^{2}}-\bar{b}^{2}\right) \frac{1}{N} \sum_{\alpha} \frac{1}{2 \pi} \int_{\infty}^{\infty}\left\langle\cdot e^{-i Q \cdot r_{\alpha}(0)} e^{i Q \cdot r_{\alpha}(t)}\right\rangle e^{-i \omega t} d t \\
\frac{d^{2} \sigma}{d \Omega d \omega}=\left(\frac{d^{2} \sigma}{d \Omega d \omega}\right)_{c o h}+\left(\frac{d^{2} \sigma}{d \Omega d \omega}\right)_{i n c}
\end{gathered}
$$


On définit habituellement la fonction de diffusion intermédiaire, $I(Q, t)$,

$$
I(Q, t)=\frac{1}{N} \sum_{\alpha} \sum_{\beta}\left\langle. e^{-i Q \cdot r_{\alpha}(0)} e^{i Q \cdot r_{\beta}(t)}\right\rangle
$$

dont on prend les transformées de Fourier suivantes, désignées respectivement comme la fonction de correlation de paires dépendante du temps, $G(\boldsymbol{r}, t)$

$$
G(\boldsymbol{r}, t)=\frac{1}{(2 \pi)^{3}} \int_{-\infty}^{\infty} I(Q, t) e^{-i Q \cdot r} d Q
$$

et la fonction de diffusion, $S(Q, \omega)$ (appelée aussi loi de diffusion)

$$
S(Q, \omega)=\frac{1}{2 \pi} \int_{-\infty}^{\infty} I(Q, t) e^{-i \omega t} d t
$$

D'après les définitions des transformées de Fourier inverses correspondantes

$$
\begin{aligned}
& I(Q, t)=\int_{-\infty}^{\infty} G(r, t) e^{i Q \cdot r} d r \\
& I(Q, t)=\int_{-\infty}^{\infty} I(Q, \omega) e^{i \omega t} d t
\end{aligned}
$$

il apparaît que $S(Q, \omega)$ est la transformée de Fourier de $G(r, t)$ par rapport à l'espace et au temps :

$$
\begin{aligned}
& S(Q, \omega)=\frac{1}{2 \pi} \int_{-\infty}^{\infty} G(r, t) e^{-i(\omega t-Q \cdot r)} d Q d t \\
& G(\boldsymbol{r}, t)=\frac{1}{(2 \pi)^{3}} L_{-\infty}^{\infty} S(Q, \omega) e^{i(\omega t-Q \cdot r)} d Q d t
\end{aligned}
$$

tandis que $I(Q, t)$ est la transformée de Fourier spatiale de $G(r, t)$ et que $S(Q, \omega)$ est la transformée de Fourier temporelle de $I(Q, t)$.

Lorsque le même diffuseur intervient aux instants 0 et $t$, on définit d'une manière analogue une fonction de diffusion intermédiaire, désignée comme incohérente, $I_{\text {inc }}(Q, t)$ :

$$
I_{i n c}(Q, t)=\frac{1}{N} \sum_{\alpha}\left\langle. e^{-i Q \cdot r_{\alpha}(0)} e^{i Q \cdot r_{\alpha}(t)}\right\rangle
$$

et une fonction de diffusion incohérente, $S_{\text {inc }}(Q, \omega)$ :

$$
S_{\text {inc }}(Q, \omega)=\frac{1}{2 \pi} \int_{\infty}^{\infty} I_{\text {inc }}(Q, t) e^{-i \omega t t} d t
$$

qui est la transformée de Fourier par rapport à l'espace et au temps de la fonction d'autocorrelation de paires dépendante du temps, $G_{s}(\boldsymbol{r}, t)$ : 


$$
\begin{aligned}
& S_{i n c}(Q, \omega)=\frac{1}{2 \pi} \int_{\infty}^{\infty} G_{s}(r, t) e^{-i(\omega t-Q \cdot r)} d Q d t \\
& G_{S}(r, t)=\frac{1}{(2 \pi)^{3}} \int_{-\infty}^{\infty} S_{i n c}(Q, \omega) e^{i(\omega t-Q . r)} d Q d t
\end{aligned}
$$

Finalement, nous écrirons :

$$
\frac{d^{2} \sigma}{d \Omega d \omega}=\frac{1}{4 \pi} \frac{k}{k_{0}}\left[\sigma_{c o h} S(Q, \omega)+\sigma_{i n c} S_{i n c}(Q, \omega)\right]
$$

Nous signalerons pour terminer qu'en spectrométrie par écho de spins, l'usage est de désigner les fonctions de diffusion intermédiaires $l(Q, t)$ et $I_{\text {inc }}(Q, t)$ par $S(Q, t)$ et $S_{i n c}(Q, t)$ respectivement.

\section{LA FONCTION DE DIFFUSION INCOHERENTE}

Nous allons maintenant nous intéresser tout particulièrement à la fonction de diffusion intermédiaire incohérente relative à un diffuseur particulier

$$
I_{\text {inc }}(Q, t)=\langle\exp [i Q \cdot r(t)] \exp [-i Q \cdot r(0)]\rangle
$$

Considérant le cas général d'un composé moléculaire (cristal moléculaire, polymère, liquide moléculaire, ...), nous allons recenser les diverses composantes du vecteur $\boldsymbol{r}(t)$ repérant sa position dans l'échantillon et nous analyserons le caractère de leurs variations au cours du temps sous l'effet des vibrations internes de la molécule ainsi que de ses déplacements d'ensemble. Après avoir précisé les hypothèses selon lesquelles la dynamique de mouvements de nature différente peut être étudiée séparément nous établirons les fonctions de diffusion correspondantes.

\subsection{Séparation des mouvements}

Le vecteur $r(t)$ repérant la position instantanée d'un noyau diffuseur peut être séparé en deux composantes de nature différente: les vibrations moléculaires internes et les déplacements d'ensemble de la molécule à laquelle il appartient. Les élongations des liaisons chimiques et les modifications des angles entre ces liaisons ont pour conséquence un déplacement $v(t)$ du noyau de sa position d'équilibre dans la molécule. Dans le même temps, par rapport à un système d'axes fixes extérieur à la molécule tel celui des axes du réseau dans le cas d'un matériau cristallin, la situation instantanée de cette position d'équilibre varie sous l'effet des déplacements d'ensemble de la molécule. Suivant la nature du matériau, ces derniers peuvent prendre des aspects différents. Les liquides combinent souvent une diffusion à longue distance des molécules avec des rotations ou des réorientations de grande amplitude autour de leurs centres de gravité. Les espèces chimiques incluses en phase solide peuvent aussi être l'objet de déplacements à longue distance, généralement par sauts successifs entre sites d'adsorption. Dans les milieux solides désordonnés on peut observer des réorientations de groupements chimiques comme la réorientation de groupements méthyle latéraux dans des polymères. Le passage entre deux formes énantiomères par l'inversion d'un cycle peut induire des déplacements importants des groupes chimiques qui y sont greffés: Dans les cristaux liquides ou les cristaux plastiques des molécules entières sont susceptibles de se réorienter. Quant aux milieux cristallins, ils sont le siège de mouvements vibrationnels coopératifs (phonons).

Lorsqu'il n'existe pas de mouvements à longue distance, nous écrirons d'une manière générale la position instantanée d'un diffuseur appartenant à une molécule sous la forme

$$
r(t)=v(t)+R(\Omega, t)+u(t)
$$


dans laquelle $v(t)$ correspond aux déplacements de la molécule dans son ensemble sous l'effet des vibrations de réseau. $\boldsymbol{R}(\Omega, t)$ repère la position d'équilibre de l'atome dans la molécule lorsque cette dernière est dans l'orientation désignée par les angles d'Euler $\Omega$. Le vecteur déplacement $\boldsymbol{u}(\mathrm{t})$ tient compte du déplacement du diffuseur sous l'effet des vibrations moléculaires internes. Dans la situation où il convient d'envisager des déplacements à longue distance (phases liquides ou espèces incluses), on utilisera le développement

$$
\boldsymbol{r}(t)=\boldsymbol{T}(t)+\boldsymbol{R}(\Omega, t)+\boldsymbol{u}(t)
$$

dans lequel $\boldsymbol{T}(\mathrm{t})$ représente la translation de la molécule dans son ensemble

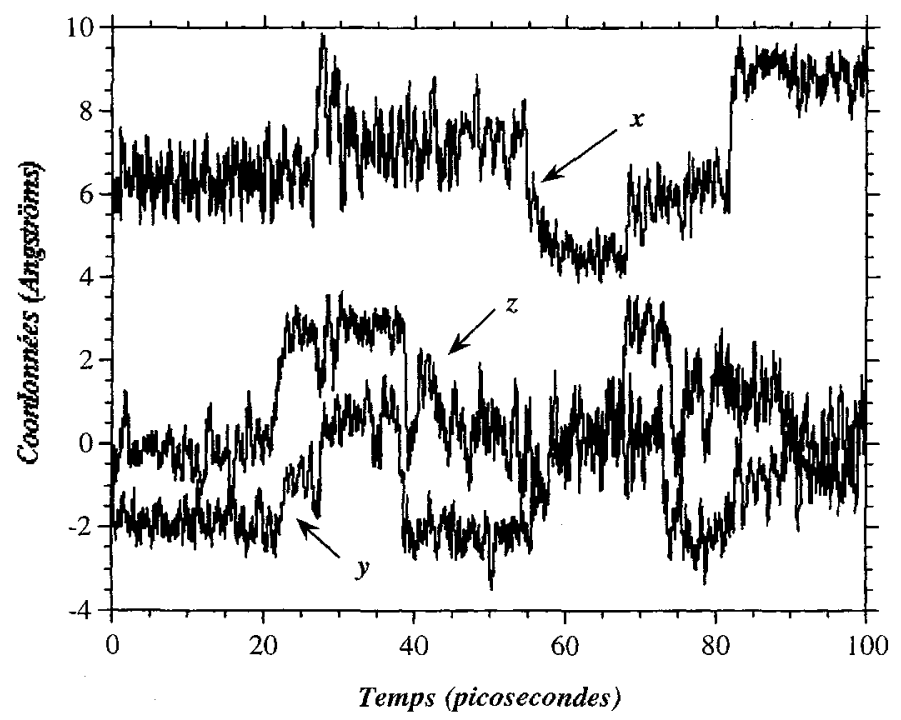

Figure 2: Trajectoire d'un des atomes d'hydrogène de la molécule d'hexaméthyléthane obtenue par simulation de dynamique moléculaire pour une température $\mathrm{T}=300 \mathrm{~K}$. Les valeurs des 3 coordonnées $(\mathrm{x}, \mathrm{y}, \mathrm{z})$ sont représentées sur une durée de 100 picosecondes. La simulation totale dure 660 picosecondes. De brusques changements d'orientation sont clairement visibles. Le temps moyen qui s'écoule entre deux réorientations est de l'ordre de $10^{-11} \mathrm{~s}$. Ce sont ces mouvements qui sont responsables de l'élargissement quasiélastique observé sur les spectres de diffusion de neutrons. Dans chacune de ses orientations d'équilibre la molécule effctue un grand nombre de vibrations et de librations (on notera que les coordonnées ne sont enregistrées que tous les 10 pas de simulation).

Un calcul exact de la fonction de diffusion incohérente qui tienne compte de tous les mouvements possibles est très difficile et à notre connaissance n'a jamais été entrepris, pour autant qu'il soit possible. Habituellement, on factorise $I_{\text {inc }}(Q, t)$ en plusieurs termes correspondant chacun à un des mouvements que nous venons d'évoquer. Il convient d'examiner les conditions de cette approximation. De notre analyse des diverses composantes du déplacement d'un noyau diffuseur, il est clair que deux échelles de temps sont en cause. Les vibrations internes des molécules sont très rapides $\left(10^{-15}-10^{-14} \mathrm{~s}\right)$ tout comme les vibrations de réseau $\left(10^{-13} \mathrm{~s}\right)$ si on fait exception des modes acoustiques de très basse fréquence. Les déplacements à longue distance de molécules en phase liquide ou d'espèces incluses sont beaucoup plus lents $\left(10^{-10}-10^{-9} \mathrm{~s}\right)$. Quant aux temps caractéristiques associés aux réorientations de groupements chimiques ou de molécules entières ils sont de l'ordre de $10^{-12}-10^{-11} \mathrm{~s}$

L'hypothèse principale est l'absence de tout couplage entre les mouvements intervenant sur des échelles de temps différentes. La figure 2 montre le résultat d'une simulation de dynamique moléculaire de la molécule d'hexaméthyléthane. Les coordonnées d'un atome d'hydrogène de l'un des groupements 
méthyles ont été suivies au cours du temps. Les réorientations de la molécule apparaissent clairement sous forme de brutales discontinuités dans la trajectoire. On remarquera aussi le grand nombre d'oscillations effectuées par la molécule sur une position d'équilibre entre deux réorientations successives. Cette observation tend à confirmer la très faible corrélation entre ces deux mouvements. Cette conclusion est renforcée par la comparaison des énergies de vibrations de l'ordre de plusieurs dizaines de meV aux faibles transferts d'énergie ( $<1$ à $2 \mathrm{meV}$ ) associés aux réorientations. De même on néglige habituellement tout couplage entre la translation et la réorientation d'une molécule. Cette seconde hypothèse, pour autant qu'elle soit commode pour les calculs est certainement moins satisfaisante. Elle suppose en particulier que les molécules se réorientent indépendamment les unes des autres et exclue les processus coopératifs. Certains résultats obtenus en dynamique moléculaire tendent à l'infïmer.

On écrira ainsi, pour un solide

$$
\begin{aligned}
I_{\text {inc }}(Q, t)= & \langle\exp (-i Q \cdot v(0)) \cdot \exp (i Q \cdot v(t))\rangle \\
& \langle\exp (-i Q \cdot \boldsymbol{u}(0)) \cdot \exp (i \boldsymbol{Q} \cdot \boldsymbol{u}(t))\rangle \\
& \langle\exp (-i \boldsymbol{Q} \cdot \boldsymbol{R}(\Omega, 0)) \cdot \exp (i \boldsymbol{Q} \cdot \boldsymbol{R}(\Omega, t))\rangle
\end{aligned}
$$

et pour un liquide

$$
\begin{aligned}
I_{\text {inc }}(Q, t)= & \langle\exp (-i Q \cdot T(0)) \cdot \exp (i Q \cdot T(t))\rangle \\
& \langle\exp (-i Q \cdot \boldsymbol{u}(0)) \cdot \exp (i \boldsymbol{Q} \cdot \boldsymbol{u}(t))\rangle \\
& \langle\exp (-i \boldsymbol{Q} \cdot \boldsymbol{R}(\Omega, 0)) \cdot \exp (i \boldsymbol{Q} \cdot \boldsymbol{R}(\Omega, t))\rangle
\end{aligned}
$$

La transformation de Fourier de ces expressions conduit aux fonctions de diffusion correspondantes qui apparaissent comme les produits de convolution

$$
\begin{aligned}
& S_{\text {inc }}(\boldsymbol{Q}, \omega)=S_{v}(\boldsymbol{Q}, \omega) \otimes S_{u}(\boldsymbol{Q}, \omega) \otimes S_{R}(Q, \omega) \\
& S_{\text {inc }}(\boldsymbol{Q}, \omega)=S_{T}(\boldsymbol{Q}, \omega) \otimes S_{u}(\boldsymbol{Q}, \omega) \otimes S_{R}(\boldsymbol{Q}, \omega)
\end{aligned}
$$

des fonctions de diffusion associées à chaque type de mouvement. Il convient d'être prudent dans l'évaluation des moyennes thermiques car les opérateurs mis en jeu ne commutent pas. Moyennant quelques corrections, $S_{T}(Q, \omega)$ et $S_{R}(Q, \omega)$ sont évaluées par la mécanique classique. On trouvera dans les ouvrages de base $[1,2]$ le calcul des termes $S_{v}(Q, \omega)$ et $S_{u}(Q, \omega)$ qui font intervenir la mécanique quantique. Nous nous bornerons à en donner une description générale. Ces fonctions de diffusion associées aux vibrations moléculaires et aux phonons peuvent être décomposées formellement en une partie élastique et une partie inélastique

$$
\left.\begin{array}{l}
S_{v}(Q, \omega)=\exp \left(-2 W_{v}(Q)\right)\left\{\delta(\omega)+S_{v}^{\text {inél }}(Q, \omega)\right\} \\
S_{u}(Q, \omega)=\exp \left(-2 W_{u}(Q)\right)\left\{\delta(\omega)+S_{u}^{\text {inél }}(Q, \omega)\right.
\end{array}\right\}
$$

Ces expressions font apparaître des termes de Debye-Waller

$$
\begin{aligned}
& 2 W_{v}(Q)=<v^{2}>Q^{2} \\
& 2 W_{u}(Q)=<u^{2}>Q^{2}
\end{aligned}
$$

dans lesquels $\left\langle v^{2}\right\rangle$ et $\left\langle u^{2}\right\rangle$ représentent les déplacements carrés moyens du diffuseur sous l'effet respectivement des vibrations internes et des vibrations et librations d'ensemble de la molécule. $S_{v}^{i n e ́ l}(Q, \omega)$ se compose d'un ensemble de raies étroites (pics de Dirac) caractéristiques des modes normaux de la molécule. Bien que ces raies s'élargissent quand elles sont convoluées avec les autres 
termes, en raison de leur localisation aux transferts d'énergie élevés, elles n'ont aucune incidence sur la partie quasiélastique des spectres. La fonction $S_{u}^{\text {inél }}(\boldsymbol{Q}, \omega)$ est liée à la densité des états de vibration du réseau. Elle contribue à l'intensité diffusée dans la région des faibles transferts d'énergies jusqu'à environ $20 \mathrm{meV}$. Elle présente des maxima pour les fréquences optiques situées en centre de zone de Brillouin et pour les modes optiques et acoustiques en limite de zone. Cependant, dans la région quasiélastique cette intensité est généralement de peu d'importance et prend la forme d'un bruit de fond pratiquement plat (figure 3)

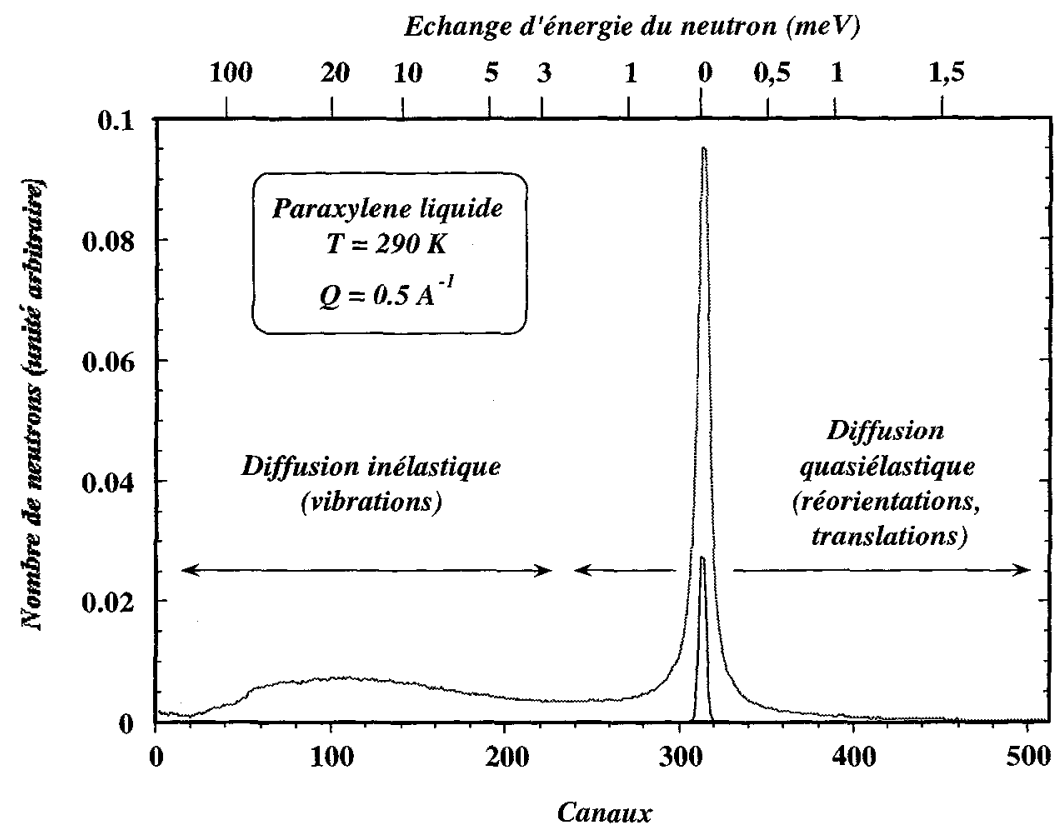

Figure 3 : Exemple de spectre de diffusion quasiélastique obtenu sur un spectromètre du type « temps de vol ». L'échelle en canaux (donc en temps) montre clairement la séparation en une région inélastique correspondant aux modes de vibrations et une région quasiélastique associées aux réorientations et aux translations. La fonction de résolution expérimentale esı représentée et permet de se rendre compte de l'élargissement du pic quasiélastique. la forme

Ces considérations nous autorisent à écrire le résultat des produits de convolutions précédents sous

$$
S_{i n c}(Q, \omega)=\exp (-2 W(Q))\left\{S_{R}(Q, \omega)+S^{i n e ́ l}(Q, \omega)\right\}
$$

pour un solide et

$$
S_{\text {inc }}(\boldsymbol{Q}, \omega)=\exp (-2 W(Q))\left\{S_{T}(\boldsymbol{Q}, \omega) \otimes S_{R}(\boldsymbol{Q}, \omega)+S^{\text {inél }}(\boldsymbol{Q}, \omega)\right\}
$$

en présence d'un désordre de translation. Le terme de Debye-Waller

$$
W(Q)=W_{v}(Q)+W_{u}(Q)
$$


tient compte de façon globale des vibrations intramoléculaires et des vibrations de réseau. Le terme inélastique global $S^{\text {inél }}(Q, \omega)$ résulte de la convolution de $S_{\nu}(Q, \omega)$ et $S_{u}(Q, \omega)$ avec $S_{R}(Q, \omega)$ ou de $S_{u}(\boldsymbol{Q}, \omega)$ avec $S_{T}(\boldsymbol{Q}, \omega) \otimes S_{R}(\boldsymbol{Q}, \omega)$. Il ne concerne qu'une très faible part de l'intensité diffusée dans la région quasiélastique et peut toujours être pris en compte sous la forme d'une densité d'état de Debye.

$S_{T}(Q, \omega)$ et $S_{R}(Q, \omega)$ sont les grandeurs fondamentales qui seront étudiées ici. Ce sont les termes quasiélastiques dont l'analyse renseigne sur les mouvements diffusifs des atomes de l'échantillon.

\subsection{Calcul des fonctions de diffusion dans l'approximation classique}

Comme nous l'avons mentionné les fonctions de diffusion rotationnelle et translationnelle de calculent par la mécanique classique. En effet les échanges d'énergie $\hbar \omega$ ainsi que les transferts de moment $\hbar Q$ mis en jeu sont tels que

$$
\begin{aligned}
& |\hbar \omega|<\frac{1}{2} k_{B} T \\
& \frac{\hbar^{2} Q^{2}}{2 m} \ll \frac{1}{2} k_{B} T
\end{aligned}
$$

où $\mathrm{M}$ est la masse du diffuseur et $\frac{1}{2} k_{B} T$ correspond à l'énergie thermique par degré de liberté. Dans ces conditions $\boldsymbol{R}(\Omega, t)$ ou $\boldsymbol{T}(t), \boldsymbol{R}(\Omega, 0)$ ou $\boldsymbol{T}(0)$ sont simplement des vecteurs $\boldsymbol{R}(t)$ et $\boldsymbol{R}(0)$ repérant les positions du noyau diffuseur aux instants respectifs $t$ et 0 . Le calcul de la fonction intermédiaire se ramène alors à un calcul de probabilités

$$
I_{i n c}(\boldsymbol{Q}, t)=\iint p(\boldsymbol{R}(0) ; \boldsymbol{R}(t)) \cdot p(\boldsymbol{R}(0)) \cdot \exp (-i \boldsymbol{Q} \cdot \boldsymbol{R}(0)) \cdot \exp (i \boldsymbol{Q} \cdot \boldsymbol{R}(t)) \cdot d \boldsymbol{R}(t) \cdot d \boldsymbol{R}(0)
$$

dans lequel $p(\boldsymbol{R}(0) ; \boldsymbol{R}(t))$ représente la probabilité qu'un atome diffuseur, situé en $\boldsymbol{R}(0)$ à l'instant initial, soit en $\boldsymbol{R}(t)$ à l'instant $t$. La distribution des probabilités à l'instant initial est décrite par $p(\boldsymbol{R}(0))$. Ces fonctions caractérisent le modèle utilisé pour décrire la dynamique de l'échantillon. En général elles dépendent de paramètres physiques (longueurs de sauts, temps caractéristiques...) qui sont déterminés par comparaison et affinement de la loi de diffusion théorique aux spectres expérimentaux.

Les lois de diffusion calculées théoriquement sont symétriques

$$
S^{c l}(Q, \omega)=S^{c l}(-Q,-\omega)
$$

et violent le principe du bilan détaillé

$$
S^{c l}(Q, \omega)=\exp \left(\frac{\hbar \omega}{k_{B} T}\right) \cdot S^{c l}(-Q,-\omega)
$$

Dans l'expression précédente, $\hbar \omega$ est le gain d'énergie $\underline{d u}$ neutron. On obtient une très bonne approximation de la fonction de diffusion réelle en posant

$$
S(Q, \omega)=\exp \left(-\frac{\hbar \omega}{2 k_{B} T}\right) S^{c l}(Q, \omega)
$$




\section{PROPRIETES GENERALES DES FONCTIONS DE DIFFUSION ROTATIONNELLE ET TRANSLATIONNELLE}

Reprenons l'expression de la fonction de diffusion intermédiaire relative à un diffuseur unique

$$
I_{\text {inc }}(\boldsymbol{Q}, t)=\iint p(\boldsymbol{R}(0) ; \boldsymbol{R}(t)) \cdot p(\boldsymbol{R}(0)) \cdot \exp (-i \boldsymbol{Q} \cdot \boldsymbol{R}(0)) \cdot \exp (i \boldsymbol{Q} \cdot \boldsymbol{R}(t)) \cdot d \boldsymbol{R}(t) \cdot d \boldsymbol{R}(0)
$$

Les corrélations éventuelles entre les positions du même diffuseur à l'instant initial $\boldsymbol{R}(0)$ et à un instant ultérieur, $\boldsymbol{R}(t)$, diminuent au cours du temps et finissent par disparâttre complètement au temps infini.

$$
p(\boldsymbol{R}(0) ; \boldsymbol{R}(\infty))=p(\boldsymbol{R}(\infty))
$$

Ainsi la moyenne précédente peut-elle être prise séparément sur les positions initiale et finale du diffuseur

$$
\begin{aligned}
I_{i n c}(\boldsymbol{Q}, \infty) & =\iint p(\boldsymbol{R}(\infty)) \cdot p(\boldsymbol{R}(0)) \cdot \exp (-i \boldsymbol{Q} \cdot \boldsymbol{R}(0)) \cdot \exp (i Q \cdot \boldsymbol{R}(\infty)) \cdot d \boldsymbol{R}(\infty) \cdot d \boldsymbol{R}(0) \\
& =\int p(\boldsymbol{R}(0)) \cdot \cdot \exp (-i \boldsymbol{Q} \cdot \boldsymbol{R}(0)) \cdot d \boldsymbol{R}(0) \cdot \int p(\boldsymbol{R}(\infty)) \cdot \exp (i \boldsymbol{Q} \cdot \boldsymbol{R}(\infty)) \cdot . d \boldsymbol{R}(\infty)
\end{aligned}
$$

Le système étant en équilibre, la distribution des noyaux diffuseurs est la même aux deux instants, de sorte que

$$
I_{\text {inc }}(\boldsymbol{Q}, \infty)=\left|\int p(\boldsymbol{R}(0)) \cdot \exp (-i Q \cdot \boldsymbol{R}(0)) \cdot d \boldsymbol{R}(0)\right|^{2}=\left|\int p(\boldsymbol{R}(\infty)) \cdot \exp (i Q \cdot \boldsymbol{R}(\infty)) \cdot d \boldsymbol{R}(\infty)\right|^{2}
$$

A un instant quelconque, il est possible de séparer formellement $I_{\text {inc }}(Q, t)$ en sa partie indépendante du temps, $I_{\text {inc }}(Q, \infty)$ et une partie dépendante du temps $I_{\text {inc }}^{\prime}(Q, t)$

$$
I_{i n c}(Q, t)=I_{i n c}(Q, \infty)+I_{i n c}^{\prime}(Q, t)
$$

En prenant la transformée de Fourier, la présence du terme constant, $I_{i n c}(Q, \infty)$ fait apparâtre dans l'expression de la fonction de diffusion incohérente une composante purement élastique $I_{\text {inc }}(Q, \infty) . \delta(\omega)$

$$
\begin{aligned}
S(Q, \omega) & =\frac{1}{2 \pi} \int_{-\infty}^{\infty} I_{i n c}(Q, \infty) \exp (-i \omega t) d t+\frac{1}{2 \pi} \int_{-\infty}^{\infty} I_{i n c}^{\prime}(Q, t) \exp (-i \omega t) d t \\
& =I_{i n c}(Q, \infty) \cdot \delta(\omega)+\frac{1}{2 \pi} \int_{\infty}^{\infty} I_{i n c}^{\prime}(Q, t) \exp (-i \omega t) d t
\end{aligned}
$$

La forme et la largeur de la seconde composante dépendent de la nature et des temps caractéristiques des mouvements des noyaux. Dans le cas très simple où $I_{\text {inc }}^{\prime}(Q, t)$ décrô̂t avec le temps de façon exponentielle, avec un seul temps caractéristique, $\tau$,

$$
\begin{array}{r}
I^{\prime}{ }_{i n c}(Q, t)=\left[I_{i n c}(Q, 0)-I_{i n c}(Q, \infty)\right] \exp \left(-\frac{t}{\tau}\right) \\
S(Q, \omega)=I_{i n c}(Q, \infty) \cdot \delta(\omega)+\left[I_{i n c}(Q, 0)-I_{i n c}(Q, \infty] \cdot \frac{1}{\pi} \cdot \frac{\tau}{1+\omega^{2} \tau^{2}}\right.
\end{array}
$$


On aboutit à une composante quasiélastique de forme lorentzienne, sous-jacente à une composante purement élastique. Si la largeur de la composante quasiélastique est directement liée aux temps caractéristiques des mouvements dont sont animés les atomes diffuseurs, l'importance de la contribution élastique est donnée par la transformée de Fourier spatiale de leur distribution d'équilibre. Elle a la dimension d'un facteur de structure et traditionnellement est désignée sous le nom de "Facteur de structure élastique incohérent". La caractéristique générale des échantillons offrant un désordre de translation est l'absence de pic élastique. Au contraire, l'existence dans les spectres expérimentaux d'une part d'intensité diffusée élastique est le signe de la présence dans l'échantillon de diffuseurs dont le mouvement est localisé dans l'espace.

La résolution d'un spectromètre est nécessairement limitée. C'est pourquoi le pic élastique n'est jamais infiniment étroit mais présente une allure triangulaire, gaussienne ou lorentzienne dont la largeur est caractéristique de l'instrument. Cette largeur définit l'échelle de temps sur laquelle les mouvements sont observables, par exemple $10^{-10} \mathrm{~s}$ pour un spectromètre à rétrodiffusion à haute résolution, $10^{-11}-10^{-}$

${ }^{12} \mathrm{~s}$ pour un spectromètre à temps de vol. Des mouvement trop lents conduisent à des élargissements trop faibles pour être observés. Inversement des mouvements trop rapides produisent des élargissements considérables et la diffusion quasiélastique prend l'allure d'un bruit de fond dans le pied des spectres. II faut toujours avoir à l'esprit l'échelle de temps accessible au spectromètre que l'on utilise avant de tirer des conclusions sur la nature des mouvements

\section{Références}

[1] Egelstaff P.A., Thermal Neutron Scattering (Academic, New York, 1971).

[2] Lovesey S.W., Theory of Neutron Scattering from Condensed Matter (Clarendon, Oxford, 1984).

[3) Squires G.L., Introduction to the Theory of Thermal Neutron Scattering (Cambridge University Press, Cambridge, 1978)

[4] Springer T., Quasielastic Neutron Scattering for the Investigation of Diffusive Motions in Solids and Liquids, Springer Tracts in Modern Physics (Springer, Berlin, 1972)

[5] Bée M., Quasielastic Neutron Scattering; Principles and Applications in Solid State Chemistry, Biology and Materials Science (Adam Hilger, Bristol, 1988) 